INPLASY

PROTOCOL

To cite: Quiroz et al.

Syndemics between cardiometabolic and infectious diseases in adults: a scoping review protocol. Inplasy protocol 202150048. doi: 10.37766/inplasy2021.5.0048

Received: 13 May 2021

Published: 13 May 2021

Corresponding author: Wilson Cañon-Montañez

wilson.canon@udea.edu.co

Author Affiliation:

Universidad de Antioquia

Support: Colombian Ministry of Sciences.

Review Stage at time of this submission: Preliminary searches.

Conflicts of interest:

None declared.

\section{Syndemics between cardiometabolic and infectious diseases in adults: a scoping review protocol}

Quiroz, SP1; Piñeros, JG2; Cañon-Montañez, W33.

Review question / Objective: What is the definition used to characterize syndemics in studies that evaluated syndemics between cardiometabolic and infectious diseases?

Condition being studied: The relationship between infectious and cardiometabolic diseases emerged several decades ago. For example, evidence on the effect of periodontal infection and cardiovascular diseases, chlamydia pneumoniae and atherosclerosis, and enterovirus and type 1 diabetes mellitus have been reported. Although this relationship has been investigated in different populations, there is a need to synthesize this evidence to identify the forms of relationship and the mechanisms established in the approach to this phenomenon (causal association, interaction or syndemics). This synthesis is gaining even more interest today due to the impact of adverse outcomes generated by the interaction between cardiometabolic diseases and the recent COVID-19 pandemic.

INPLASY registration number: This protocol was registered with the International Platform of Registered Systematic Review and Meta-Analysis Protocols (INPLASY) on 13 May 2021 and was last updated on 13 May 2021 (registration number INPLASY202150048).

\section{INTRODUCTION}

Review question / Objective: What is the definition used to characterize syndemics in studies that evaluated syndemics between cardiometabolic and infectious diseases?
Condition being studied: The relationship between infectious and cardiometabolic diseases emerged several decades ago. For example, evidence on the effect of periodontal infection and cardiovascular diseases, chlamydia pneumoniae and atherosclerosis, and enterovirus and type 1 diabetes mellitus have been reported. 
Although this relationship has been investigated in different populations, there is a need to synthesize this evidence to identify the forms of relationship and the mechanisms established in the approach to this phenomenon (causal association, interaction or syndemics). This synthesis is gaining even more interest today due to the impact of adverse outcomes generated by the interaction between cardiometabolic diseases and the recent COVID-19 pandemic.

\section{METHODS}

Search strategy: The search will be carried out in the following databases: MEDLINE, EMBASE, Latin American Caribbean Health Sciences Literature (LILACS), Cochrane CENTRAL, Web of Science, and Scopus. The combination of the following descriptors, terms and keywords will be used for search strategy: syndemics, convergence diseases, coexistent condition, infection diseases, cardiometabolic diseases, noncommunicable diseases.

Participant or population: Adults of both sexes. Studies in people with disabilities and pregnant women will be excluded.

Intervention: Cardiometabolic and infectious diseases.

Comparator: Not applicable.

Study designs to be included: Crosssectional, case-control, and cohort studies.

Eligibility criteria: Observational studies and/or studies with data analytics (machine learning, big data) that analyze the relationship (association, interaction, and syndemics) between cardiometabolic diseases, COVID-19 and other infectious diseases will be included. Review studies, case reports, case series, editorials and letters to the editor will not be included. Duplicate studies will be eliminated.

Information sources: Electronic databases: Medline, Embase, Lilacs, Cochrane CENTRAL, Web of Science, Scopus. To identify additional studies, gray literature will also be searched. There will be no restrictions on language and year of publication.

Main outcome(s): Description of definitions used to characterize syndemics in studies that assessed syndemics between cardiometabolic and infectious diseases.

Data management: The following variables will be extracted from studies: author, year of publication, study design, sample, mean age, diagnostic criteria used for each disease, other reported comorbidities, and inclusion and exclusion criteria for participants, types of proposed relationship (linear, bidirectional, causal, interaction, syndemics), relationship evaluation methods.

Quality assessment / Risk of bias analysis: The methodological quality of studies will be assessed using the National Institutes of Health (NIH) tools for observational studies.

Strategy of data synthesis: The description of the definitions used to define the syndemics between cardiometabolic and infectious diseases will be done narratively without the inclusion of an analysis of the data.

Subgroup analysis: Not applicable.

Sensitivity analysis: Not applicable.

Country(ies) involved: Colombia.

Keywords: Syndemics; cardiometabolic diseases; infectious diseases.

Dissemination plans: The journal for the publication of the review manuscript has not yet been determined.

Contributions of each author:

Author 1 - Silvia Patricia Quiroz Mena.

Email: silvia.quiroz@udea.edu.co

Author 2 - Juan Gabriel Piñeros Jimenez.

Email: juan.pineros@udea.edu.co

Author 3 - Wilson Cañon-Montañez.

Email: wilson.canon@udea.edu.co 